\title{
Alterstice
}

Revue internationale de la recherche interculturelle

International Journal of Intercultural Research

Revista International de la Investigacion Intercultural

\section{Phénoménologie du Merveilleux, sous la direction de Schallum Pierre}

\section{Chantale Pilon}

Volume 2, numéro 2, 2012

URI : https://id.erudit.org/iderudit/1077571ar

DOI : https://doi.org/10.7202/1077571ar

Aller au sommaire du numéro

Éditeur(s)

Alterstice

ISSN

1923-919X (numérique)

Découvrir la revue

Citer ce compte rendu

Pilon, C. (2012). Compte rendu de [Phénoménologie du Merveilleux, sous la

direction de Schallum Pierre]. Alterstice, 2(2), 121-124.

https://doi.org/10.7202/1077571ar d'utilisation que vous pouvez consulter en ligne.

https://apropos.erudit.org/fr/usagers/politique-dutilisation/ 


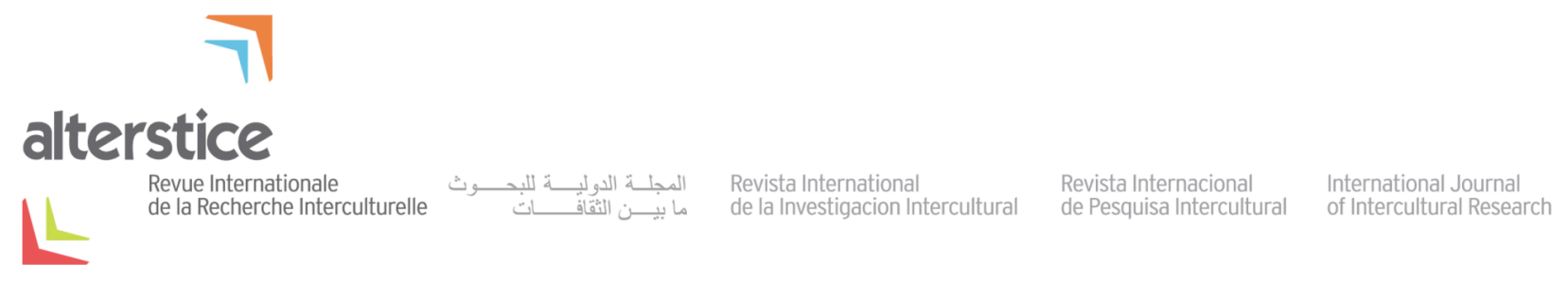

NOTE DE LECTURE

\title{
Phénoménologie du Merveilleux, sous la direction de Schallum Pierre
}

\author{
Chantale Pilon $^{1}$
}

\author{
Rattachement de l'auteure \\ ${ }^{1}$ Université Laval, Québec, Canada \\ Correspondance \\ chantale.pilon.1@ulaval.ca \\ Référence de l'ouvrage :
}

Pierre, S. (dir.). (2011). Phénoménologie du Merveilleux. Presse de I'Université du Québec, 224 p.

\section{Pour citer cet article :}

Pilon, C. (2012). Phénoménologie du Merveilleux [Note de lecture]. Alterstice, 2(2), 121-124.

Cet ouvrage tire son origine d'un colloque sur le merveilleux tenu au Musée de la civilisation de Québec en octobre 2010, dans le cadre de la première édition du festival littéraire Québec en toutes lettres de l'Institut canadien de Québec. Le livre se présente comme un ensemble polyphonique invitant à mieux percevoir la réalité fondamentale du merveilleux. Les différentes communications rassemblées dans ce recueil témoignent d'ailleurs fort bien de la richesse et de la complexité du thème, où se rencontrent notamment la littérature, l'anthropologie, les sciences religieuses, le cinéma et la philosophie. L'ouvrage constitue une lecture dialogique riche et parfois passionnante qui montre que la notion de merveilleux va droit au cœur de l'expérience humaine. La présence du merveilleux dans les manifestations de l'humain ouvre la voie à la lecture phénoménologique qui sert de fil directeur à l'ouvrage. Le caractère fondamental et universel de ce thème suffit d'ailleurs à justifier l'intérêt d'une approche interdisciplinaire.

S'intéresser au merveilleux, c'est prendre à revers les problématiques incontournables du nihilisme moderne, du réductionnisme positiviste et de l'individualisme possessif qui gangrènent le contexte de construction socioéconomique occidental. Le premier mérite de ce livre est donc de mettre à l'avant-scène la thématique trop souvent négligée et sous-exploitée du merveilleux. Volontiers associé au déraisonnable, à la fantaisie ou au futile, le concept de merveilleux souffre, dans la culture dominante, d'une sévère déconsidération. Pourtant, la relation que l’homme entretient avec le merveilleux semble intrinsèquement liée aux fondements anthropologiques de la socialité.

Certes, il n'y a pas d'émerveillement sans étonnement. Ces termes paraissent indissociables et sont, le plus souvent, traités de concert par les différents auteurs. Dans le premier texte, Thomas de Koninck s'inspire de la 
quête de sens du Petit Prince afin de proposer une réflexion très intéressante sur l'émerveillement. Ce concept est défini comme éveil de la conscience à la profondeur, à l'amplitude et au mystère du monde. Il est le « point de départ indépassable " (p. 5) qui marque le passage d'un monde où tout va de soi à un monde où rien ne va de soi (p. 6). Bref, il dévoile l'extraordinaire richesse du monde. L'auteur note que l'étonnement se lie à l'espérance, dont il partage la structure. Il est espoir de rencontre, de découverte, de compréhension. II possède une temporalité propre qui, en commandant un moment d'arrêt, contrarie la distraction frénétique caractéristique de la modernité. L'éveil de la conscience affective à la beauté du monde permet de l'investir, de s'y attacher et de construire du sens. En cela, comme le précise l'auteur, « la planète qui compte pour nous est celle dont on a découvert la beauté, l'universel, la fragilité et la puissance de la vie, la tristesse, le désenchantement, l'insensé, la joie, l'amour, la vie du sens se construisant dans une approximation permanente » (p. 10). Présenté sous cet angle, l'appel au merveilleux déjouerait le mal qu'Hannah Arendt avait clairement su identifier : non pas la perte de soi dans le monde, mais la perte de notre lien avec le monde.

Par le biais d'une étude des genres littéraires, Carolina Ferrer montre l'élasticité et l'ambiguïté des catégories usuelles au travers de l'étude de trois types de merveilleux qui dominent la scène littéraire hispano-américaine : le fantastique, le réel merveilleux et le réalisme magique. Dans son analyse des fonctions classificatoires, l'auteur note d'intéressants contrastes entre les critiques et les écrivains, mais aussi entre les écrivains eux-mêmes. II semble ainsi que le merveilleux ne consente pas aisément à se laisser réduire. Cette difficulté relève de l'imbrication inextricable du concept dans l'histoire et la géographie hispano-américaines. On apprend ainsi que le "boom du roman hispano-américain " (p. 29) se développe conjointement avec les principaux événements qui ponctuent le chemin menant de la révolution cubaine aux dictatures militaires du cône sud du continent. Ici, le recours à la fiction devient un mode social de résistance face à l'agresseur, d'une part, sous la forme d'une littérature testimoniale qui « se construit à partir d'une approche réaliste et a pour but de dénoncer l'horreur vécue sous la dictature » (p. 34) face au discours monolithique dictatorial et, d'autre part, en prenant appui sur une forme de littérature qui concerne " la perception de la réalité, sur le sens et l'absence de sens de cette réalité » (p. 29).

Brian Monast, pour sa part, attaque directement le réductionnisme positiviste qui domine la tradition occidentale et dénonce sa prétention au monopole de la connaissance valide. II ne s'agit cependant pas, pour lui, de dévaloriser la connaissance dite objective. L'auteur s'emploie à démontrer qu'il existe, en marge de cette dernière, une connaissance subjective qui puise à même la richesse de l'être réel. Il soutient que ces deux types de connaissance ne sont pas antinomiques, mais qu'elles concernent plutôt des dimensions radicalement différentes de l'expérience. La critique s'adresse au caractère hégémonique de la connaissance objective, qui tend à négliger voire à invalider tout ce qui déborde du cadre structurel qu'elle implique et la détermine en retour. Brian Monast tente de démontrer que la hiérarchisation de la valeur des savoirs qui discrédite la connaissance subjective et voile le merveilleux ne relève pas d'une subordination effective, mais d'une déformation conceptuelle.

Le texte de Billy Vatcher s'intéresse à l'étonnement comme " désir de savoir 》 et aux fonctions pédagogiques de l'art universel de la magie. Selon lui, la rencontre de l'ignorance ne survient jamais seule. Elle est tout aussi surprise de découvrir ce qu'elle ignore que de se découvrir ignorante. C'est en ce sens que l'étonnement permet le passage de la double ignorance (ignorer qu'on ignore) à la simple ignorance (savoir qu'on ignore) et que l'auteur peut dire que l'étonnement enseigne de lui-même. De ce double choc naît le désir d'apprendre, par l'éveil non pas d'un intérêt fugace mais d'une curiosité soutenue. C'est d'ailleurs cet enracinement durable de l'émoi qui distingue la surprise de l'étonnement. Ce texte offre des remarques fertiles sur les concepts d'étonnement et d'illusion. L'auteur se tourne ensuite vers l'art de la magie, qu'il nous présente comme un art collaboratif où le spectateur est impliqué dans la performance. Parce qu'il s'appuie sur l'étonnement, cet art bouleverse les conventions et les convictions dans lesquelles se réfugie la conscience et, ce faisant, encourage son réveil. C'est donc en provoquant la rencontre du merveilleux que la magie élargirait le champ des possibles de l'expérience humaine. L'illusion dresse un pont entre le monde externe réaliste et le monde interne autistique. C'est notamment en tant qu'expérience bouleversante et art de l'enchantement que la magie participe à la quête de connaissance.

Jean Désy s'appuie sur son expérience avec les communautés inuites du Nunavik pour d'une part attirer notre attention sur la place centrale accordée au merveilleux dans la culture traditionnelle nordique et d'autre part nous présenter les enjeux liés au métissage culturel. La brusque mutation sociétale qui marque le passage d'une vision 
sacrée du monde à une vision pragmatique et utilitariste conforme aux diktats des technosciences tend ici vers l'acculturation. Selon l'auteur, l'expérience du merveilleux se présente comme un mode d'enracinement et de liaison permettant d'habiter le monde, de le faire sien et de le rendre signifiant. Si l'étonnement est espoir, l'auteur soutient que la déconsidération de l'attachement atavique à l'univers du merveilleux fait place au désespoir. Jean Désy met en évidence les dégâts humains causés par les transformations qui touchent en profondeur les sociétés nordiques modernes et démontre que ces transformations procèdent de l'évincement brutal de la dimension merveilleuse de l'expérience.

Alors que la logique technoscientifique qui accompagne la modernité occidentale tend à déprécier le merveilleux, Éric Crégheur nous rappelle la riche diversité du christianisme des premiers siècles. Pour l'essentiel, il s'agit d'un commentaire exégétique des Livres de léou qui, d'ailleurs, concerne peut-être davantage les exégètes. Ces livres se présentent comme des artefacts culturels. II s'agit de témoignages manifestes de la fertilité de l'imagination des gnostiques. Dans la perspective qui nous est ici présentée, le merveilleux est à entendre comme la finalité de l'existence humaine, un trésor auquel l'âme ne peut accéder qu'au terme d'un épineux périple.

En ce sens, la tradition gnostique rejoint la majorité des traditions du monde. Contrairement à la société occidentale contemporaine, ces dernières ont choisi de conserver la place du merveilleux. Sylvie Poirier s'appuie sur son expérience auprès des aborigènes australiens pour questionner la place du merveilleux dans les sociétés où le rêve est considéré comme une réalité valide et comme une extension du réel. Dans ces sociétés, la relation au merveilleux détermine la façon dont l'homme pense et construit son rapport au monde, aux autres et à lui-même. L'auteur soutient que le rêve constitue un concept clé permettant d'accéder aux principes ontologiques et épistémologiques qui structurent ces sociétés. II permet de mieux saisir "le rapport au réel, le statut de l'imaginaire, le champ de l'action, de la cognition et de la perception humaine, ainsi que toute la question du rapport entre le visible et l'invisible et la nature des frontières ontologiques entre l'humain et le non humain " (p. 10). La conception aborigène, fondée sur le pouvoir d'évocation du rêve, oppose aux conceptions réifiées une approche dynamique qui ne limite pas l'expérience au monde perceptible, immuable et prévisible. Ce texte éclairant ouvre des perspectives fascinantes sur les transferts de la pensée et de l'expérience.

Olivier Ducharme fait appel à l'œuvre poétique et cinématographique de Pierre Perrault pour nous présenter une conception du réel "comme merveille plutôt que la merveille comme réalité » (p. 159). C'est par le biais d'un périple à la découverte de l'inconnu du territoire québécois que le cinéaste entend développer ce concept. Si « pour Perrault, la réalité dans laquelle nous vivons est toute entière poésie » (p. 166), cette réalité merveilleuse ne se donne cependant pas d'emblée. Elle passe par la capacité de description et de nomination et commande de s'extraire des systèmes épistémologiques conventionnels pour s'ouvrir à une réelle rencontre avec l'inconnu. II s'agit de déjouer la tentation de ramener l'inconnu au connu. Selon lui, cette réalité devient réelle non pas par la représentation de ceux qui la vivent, mais par la présentation de la réalité vécue des hommes. Aussi, afin d'échapper aux stratégies représentationalistes, Perrault opère un renversement. La conception de la réalité qu'il propose ne se situe plus à l'extérieur de l'homme, mais se trouve introjectée en lui et se traduit par l'action. Or c'est en réinscrivant la recherche identitaire dans la connaissance du territoire au travers du langage des gens qui vivent le territoire que Perrault serait parvenu à mettre en place une éthique du merveilleux reposant sur la rencontre et l'intercession du discours. L'ouverture à la parole devient ici une ouverture à la vita activa et, ce faisant, permet le déploiement des potentialités humaines.

Schallum Pierre s'intéresse à la parenté entre le merveilleux et les droits de l'homme par le biais d'une étude sur la relation qui s'établit entre la conception d'André Breton et celle d'Aimé Césaire. Le merveilleux surréaliste, qu'André Breton situe entre rêve et réalité, est lié à la question de l'inattendu, à la rencontre fortuite d'éléments étrangers et s'associe à une beauté convulsive. L'imagination, redéfinie « en tant que faculté réalisante » (p. 181), se distingue de la phantasia grecque. Cette mise en exergue de l'imagination doit permettre de réconcilier l'irrationnel et le rationnel, de relativiser l'emprise de la "raison logicienne » (p. 181) et de "remuer la sensibilité humaine » (p. 181). Le désir de penser librement conduit le mouvement à s'émanciper des idéologies politiques. Aimé Césaire, pour sa part, développe une pensée de la " Négritude » où l'idée d'un merveilleux individuel s'insère dans l'avènement du « je " prodigieux. La facture ouvertement dénonciatrice des poèmes de l'auteur annonce la révolution. La Négritude vise la dénonciation de l'esclavage, la réappropriation de "l'héritage des déportés » 
(p. 188), la prise « de conscience et l'acception de soi » nécessaires à la reconquête de la dignité. Elle ouvre sur le miracle que constituent le refus de sombrer dans l'anonymat et la revendication à l'humanité. La Négritude, parce qu'elle illustre la lutte contre la discrimination, est de n'importe qu'elle couleur. C'est par la rencontre d'André Breton avec la poésie « de combat et de la liberté» (p. 196) césarienne que s'opère la réintroduction de la dimension sociopolitique « de la Révolution universelle» (p. 198) surréaliste.

À priori, il peut paraître difficile de percevoir un lien clair entre les différents textes, dont les objets sont souvent hétérogènes et relèvent de disciplines habituellement hermétiques les unes aux autres. Cependant, la présentation des diverses facettes du merveilleux fait ressortir une critique souterraine de l'ethnocentrisme de la culture dominante, qui tend à imposer son système du savoir. D'abord, parce que ce dernier plie la vie pour la restreindre à une structure rigide. Ensuite, parce qu'il échoue à établir une «vision du monde » (au sens de Rickert) qui donne accès à la partie du monde qui nous concerne, c'est-à-dire celle qui traite de la dimension humaine de l'expérience et permet d'orienter et d'informer notre prise de position par rapport au monde. Enfin, parce qu'elle considère tout autre mode de recherche du savoir comme une infraction aux valeurs de vérité. Elle fait ainsi écran à d'autres traditions historiques et culturelles. La "raison logicienne", parce qu'elle s'appuie sur la stratégie représentationaliste, pose le monde comme extérieur à nous. Elle a pour conséquence majeure de briser notre lien avec le monde. Car si le monde était vraiment tel qu'il est, immuable, indépendant de nous, il n'existerait pas de lien nous permettant de le connaître. Or il faut avoir un lien avec le monde réel, croire d'abord qu'il est notre monde pour envisager d'autres mondes possibles, dénoncer les injustices du monde présent et concevoir de nouveaux modes d'existence. Bref, la critique dénonce l'extension de la " raison logicienne » à tous les domaines de la vie, au mépris de tout contenu, de toute qualité et de toute différence dans l'être. C'est donc à une tentative de décolonisation de la pensée par la remise en cause de la légitimité de ce règne que nous convie la pluralité des voix présentées dans cet ouvrage.

La panoplie des textes qui constitue l'ouvrage pourrait d'ailleurs être regroupée en trois catégories: le « désir de savoir » qui lie l'étonnement à la conscience affective, les alternatives à la " raison logicienne » occidentale et enfin les dimensions éthiques et sociopolitiques que soulève la notion de merveilleux. Les auteurs ayant collaboré à cet ouvrage ont tenté de dépasser les frontières conventionnelles de leur champ de compétence pour ouvrir le dialogue et proposer de nouvelles hypothèses. Cette excursion permet la rencontre des colorations régionales du concept et du type de société que ces formes d'appropriation conditionnent. Dans certains textes, le concept de merveilleux se fait plus discret. Cependant, la force de l'ouvrage réside dans la mise en relation des textes, dans l'odyssée à laquelle est convié le lecteur à travers une lecture bigarrée et interdisciplinaire qui défie les limites de l'espace et du temps. Et c'est alors que le caractère essentiel du concept pour la compréhension des phénomènes sociaux apparaît pleinement, en filigrane, et qu'il jaillit de la mise en commun des diverses réflexions.

Alterstice - Revue Internationale de la Recherche Interculturelle, vol. 2, $n^{\circ} 2$ 